\title{
Modeling Segregation in Modulated Granular Flow
}

\author{
Richard M. Lueptow ${ }^{1,2, *}$, Zhekai Deng ${ }^{2}$, Hongyi Xiao ${ }^{1}$, and Paul B. Umbanhowar ${ }^{1}$ \\ ${ }^{1}$ Department of Mechanical Engineering, Northwestern University, Evanston, IL 60208, USA \\ ${ }^{2}$ Department of Chemical and Biological Engineering, Northwestern University, Evanston, IL 60208, USA
}

\begin{abstract}
We consider unsteady segregating granular flows, focusing on stratification in bidisperse bounded heap flow. Experiments indicate that periodically changing the feed rate of particles falling onto the upstream portion of the heap results in a stratified segregation much like that which occurs at low feed rates, but with more regular stratified layers of large and small particles and a higher overall feed flow rate. Experiments clarify how a front of large particle at a high feed rate deposits a layer of large particles that is subsequently covered over by a layer of small particles during a low feed rate, thus generating layers of large and small particles. The stratification can be modelled using a version of an advection-segregationdiffusion equation with a segregation velocity. Simply repeatedly switching the model from a low volume flow rate to a high volume flow rate instantaneously along the entire length of the flowing layer results in stratification patterns that are similar to those observed in experiments. Using a modulated feed rate offers the potential to intentionally create extended layers of bidisperse granular materials to enhance the effective degree of mixing of the deposited materials at heap length scales.
\end{abstract}

\section{Introduction}

Flowing granular materials can segregate due to particle size or density differences [1-3] to form many different pattern including stratified layers of particles [4-7]. The degree of segregation and nature of the segregation pattern depends on the flow conditions and the differences between particle species.

Previous research on segregating granular materials has focused largely on steady feed rates [5, 7]. However, here we consider the impact of modulating the feed rate in order to intentionally manipulate the segregation to generate a stratified segregation pattern, both experimentally and based on the application of an advection-segregation-diffusion model that has been successfully implemented in steady segregating flows in quasi-2D bounded heaps. We will show that by controlling the feed rate of mixed size bidisperse particles, the segregation can be controlled so that wellordered stratification of the small and large particles is achieved, as shown in Fig. 1. Such stratification may be useful in providing a better overall homogeneity at scales larger than the stratification than can be achieved without stratification for application in industrial situations such as filling a hopper.

\section{Experiments}

We consider flow in a quasi-2D, one-sided bounded heap, shown in Fig. 1. Well-mixed size bidisperse spherical glass particles (confirmed by direct sampling) are fed from a screw feeder into the system at the upper left corner and flow down the slope in a thin layer, segregating as they flow until they reach the bounding wall at the right side. The surface of the heap rises as particles fill the container.

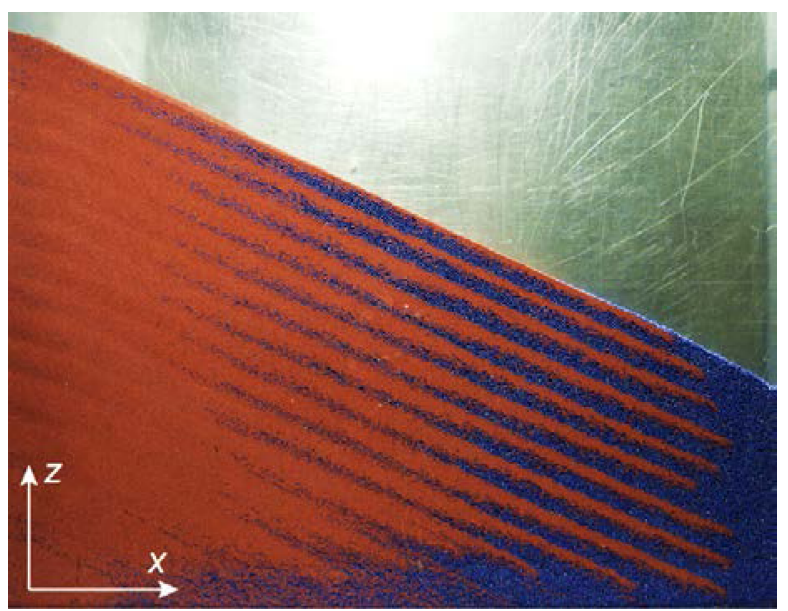

Fig. 1. A stratified pattern (enhanced contrast) generated by feeding a size bidisperse mixture $(0.05 \mathrm{~cm}$ red particles and $0.20 \mathrm{~cm}$ blue particles) onto a quasi-2D bounded heap at alternating feed rates of $q_{f}=37.0 \mathrm{~cm}^{2} / \mathrm{s}$ for $t_{f}=3 \mathrm{~s}$ and $q_{s}=0.4$ $\mathrm{cm}^{2} / \mathrm{s}$ for $t_{s}=62 \mathrm{~s}$.

For constant feed rates, the particles form irregular stratified layers at low feed rates, but segregate completely into two distinct regions with small particles upstream and large particles downstream for moderate flow rates [7]. At high enough flow rates, the particles remain mixed [7], but such high flow rates are sometimes difficult to accomplish in practical situations.

\footnotetext{
* Corresponding author: r-lueptow@northwestern.edu
} 
On the other hand, if a stratified pattern can be forced to occur, the particles are essentially mixed at the length scale of the container. It is this situation, an example of which is shown in Fig. 1, that we investigate here.

\subsection{Apparatus}

The experimental apparatus consists of a pair of parallel vertical rectangular plates - an aluminum back wall and a glass front wall for visualization. Spacers between the plates fix the gap at either $\mathrm{T}=1.27 \mathrm{~cm}$ or $\mathrm{T}=1.5 \mathrm{~cm}$ and the width at $\mathrm{W}=69 \mathrm{~cm}$, comparable in length to the flowing layer length in small industrial scale silos, as described previously [7]. Mixed glass particles are dropped vertically into the gap between the front and back walls near the left bounding wall. An auger feeder is used to feed the particles at alternating 2D flow rates: a fast flow rate $q_{f}$ for duration $t_{f}$ and a slow flow rate $q_{s}$ for duration $t_{s}$. We considered mixtures of 1) $0.050 \pm 0.004 \mathrm{~cm}$ particles and $0.200 \pm 0.007 \mathrm{~cm}$ particles; and 2) $0.118 \pm 0.007 \mathrm{~cm}$ and $0.295 \pm 0.004 \mathrm{~cm}$ particles.

Videos of the entire bed of particles are recorded at 60 frames/s with a pixel corresponding to slightly less than 1 particle diameter $(\sim 0.1 \mathrm{~cm})$ during each experiment. The free surface of the flowing layer of particles is identified by locating the transition point of the image intensity in each pixel column of the image. A time average over $0.1 \mathrm{~s}$ (6 video frames) and a space average over $0.5 \mathrm{~cm}$ are applied to reduce noise in the data.

\subsection{Results}

A typical experimental result in which the feed rate is modulated so it alternates between fast and slow values is shown in Fig. 1 for an equal volume mixture of small red particles and large blue particles. As a result of the feed flow modulation, layers of small and large particles appear. If the flow is not modulated, but operates at the same average flow rate, irregular stratification occurs [7]. Under conditions of higher flow rates, feed flow modulation results in stratification, while an equivalent constant feed rate results in a completely segregated pattern in which the small red particles deposit on the fixed bed upstream of the large blue particles resulting in a distinct vertical boundary between the red and blue particles [7]. Though completely mixed particles is typically optimal, in many applications layers of particles like that shown in Fig. 1 is preferable to completely segregated particles.

To clarify the nature of the segregation, we consider first the single transition that occurs when the flow rate changes from low to high or from high to low. In particular, we track the time evolution (time dependence) of the surface of the flowing layer at different locations along the length of the flowing layer as the change in flow rate propagates along the length of the flowing layer.

Consider the results shown in Fig. 2(a) for the transition from a low flow rate to a high flow rate at time $t=5 \mathrm{~s}$. In this figure, each curve represents the height $z h$ of the surface of the flowing layer at each of 16 locations along the length of the flowing layer as a function of time. Thus, each solid curve represents the time evolution of the surface height: the top curve corresponds to the position furthest upstream and the lowest curve corresponds to the position furthest downstream. The slope of the curve represents the velocity at which the surface is rising at that specific streamwise location. Note that if the local flow is not changing, the curves would be straight lines indicating a steady rise velocity. A change in the flow rate and associated rise velocity of the heap surface is evident as a change in the slope of the curve.
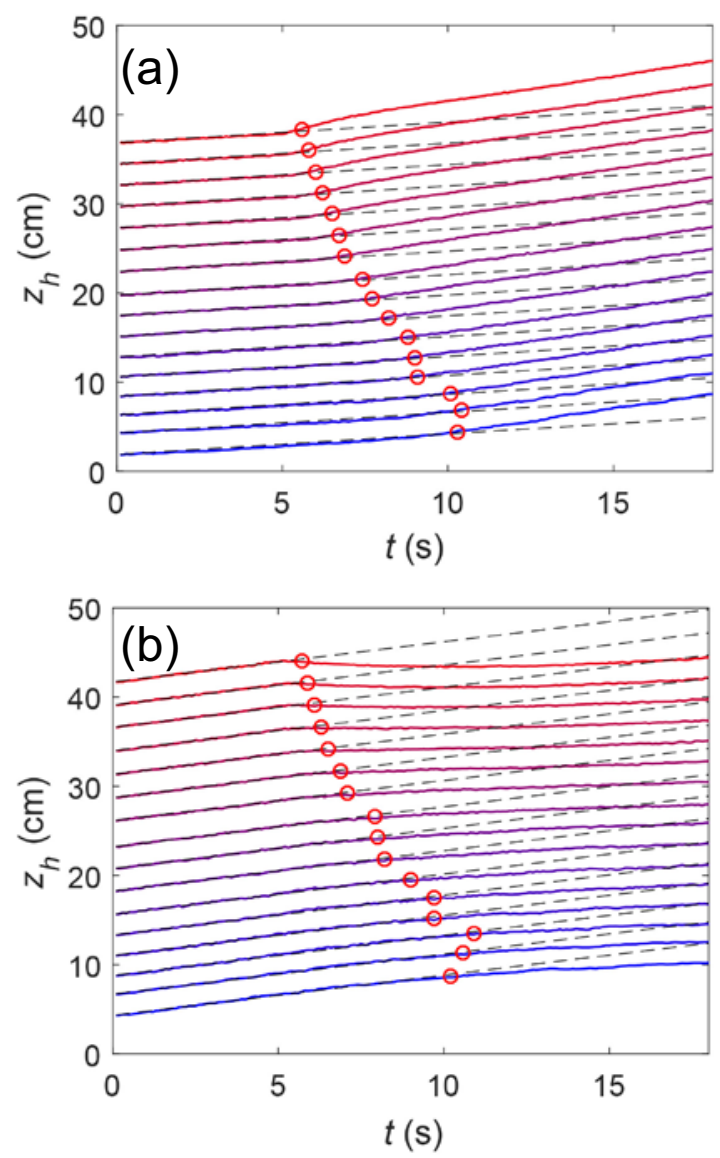

Fig. 2: The time evolution of surface height (solid lines) at various horizontal positions (from $x=8 \mathrm{~cm}$ [red] to $x=68 \mathrm{~cm}$ [blue] with interval $\Delta x=0.04 \mathrm{~m}$ ) using the bidisperse mixture $(0.118 \mathrm{~cm}$ and $0.295 \mathrm{~cm}$ particles) during (a) a single flow rate transition from $q_{s}=15.9 \mathrm{~cm}^{2} / \mathrm{s}$ to $q_{f}=37.2 \mathrm{~cm}^{2} / \mathrm{s}$ at $t=5 \mathrm{~s}$ and (b) a single flow rate transition from $q_{f}=31.1 \mathrm{~cm}^{2} / \mathrm{s}$ to $q_{s}=12.4$ $\mathrm{cm}^{2} / \mathrm{s}$ at $t=5 \mathrm{~s}$. Dashed lines depict the surface heights that would have occurred based on the initial rise velocity. The red circles mark where $z_{h}$ deviates from its expected value (based on the initial rise velocity) by more than one large particle diameter.

At the most upstream position (uppermost curve) the vertical position of the flowing layer responds almost immediately to an increase in the flow rate at $t=5 \mathrm{~s}$ with a change in slope at the point marked with a circle. The deviation between the uppermost solid curve and the dashed line demonstrates how much faster the surface at this point is rising after the flow rate increases. The 
change in flow rate does not propagate to the most downstream measurement location until about 5 seconds later, as indicated by the circle marking the change in slope of the lowest curve. Based on the circles marking the flow rate transition at all the streamwise locations, it is clear that the change in flow rate propagates downstream and that it decelerates as it travels downstream. This is not unexpected, given that a steady feed flow rate results in a flow rate that decreases to zero at the end wall of the bounded heap.

The surface height at various streamwise locations as the feed flow rate transitions from a high rate to a low rate at time $t=5 \mathrm{~s}$ is shown in Fig. 2(b). In this case the slope of the curves decreases when the transition in the flow rate reaches any particular streamwise location. Again the transition is almost immediate at the most upstream position (uppermost curve), but delayed by about $5 \mathrm{~s}$ at the most downstream location (lowermost curve).
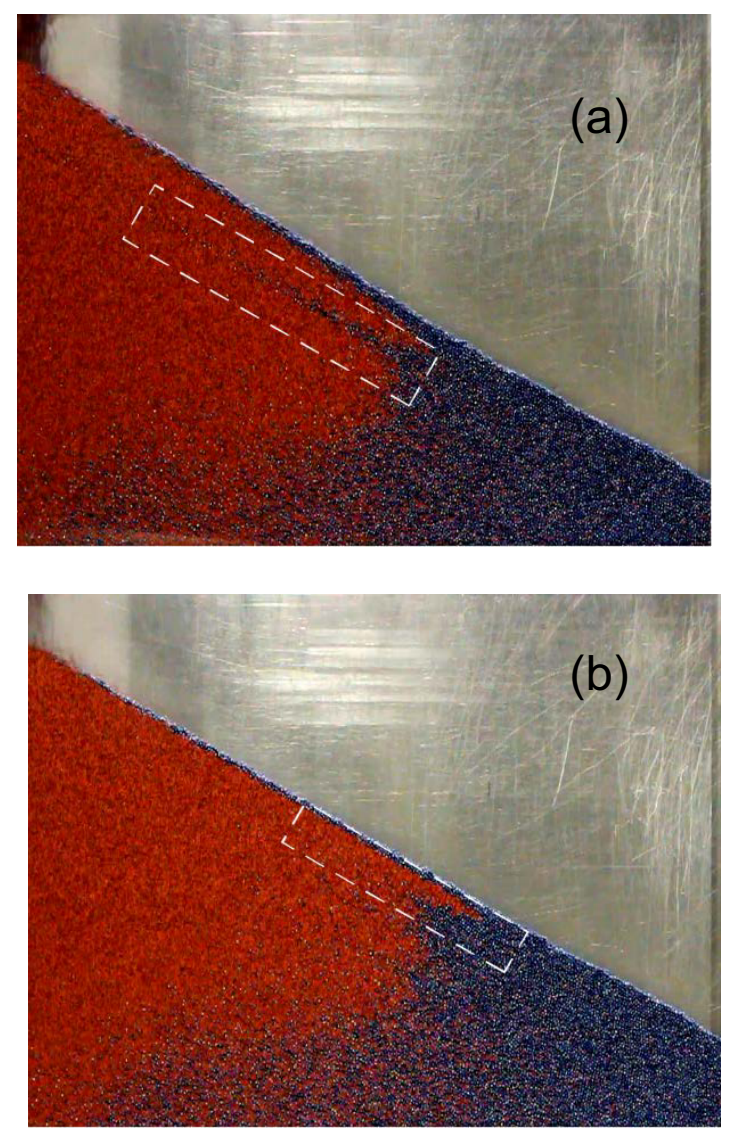

Fig. 3: Snapshots with enhanced contrast of instantaneous segregation patterns of a bidisperse mixture $(0.118 \mathrm{~cm}$ red and $0.295 \mathrm{~cm}$ blue particles) taken at $\sim 10 \mathrm{~s}$ after the transitions for (a) a single flow rate transition from $q_{s}=15.9 \mathrm{~cm}^{2} / \mathrm{s}$ to $q_{f}=37.2$ $\mathrm{cm}^{2} / \mathrm{s}$ and (b) a single flow rate transition from $q_{f}=31.1 \mathrm{~cm}^{2} / \mathrm{s}$ to $q_{s}=12.4 \mathrm{~cm}^{2} / \mathrm{s}$.

The segregation patterns that occur during a single flow rate transition are also instructive. Fig. 3(a) shows a large particle layer (blue) extending upstream into the small particle region $\sim 10 \mathrm{~s}$ after a transition from a low to a high feed flow rate. This is a result of a wedge of material deposited after the transition from the low flow rate to the high flow rate propagating downstream. The large particles accumulate at the front of the faster flowing material much like described previously for single avalanches $[8,9]$, and some are buried as the high speed flow surges downstream, thus forming the blue layer.

In Fig. 3(b), a small particle layer (red) extends into the large particle region (blue) $\sim 10 \mathrm{~s}$ after the transition from a low flow rate to a high flow rate. From Fig. 2(b), it is clear that the surface height in the upstream positions actually decreases during the first few seconds of the transition, which leads to the previously deposited small particles being carried downstream with the slower flowing material. This also means that the newly added particles are not deposited upstream, but are carried downstream. Together this extends the small particle layer further into the large particle region.

\section{Model}

A model based on the advection-diffusion equation with an additional term for segregation has been successful for modeling steady, developing, and transient granular flows of size segregating and density segregating materials [10-14]:

$$
\frac{\partial c_{i}}{\partial t}+\underbrace{\frac{\partial\left(u c_{i}\right)}{\partial x}+\frac{\partial\left(w c_{i}\right)}{\partial z}}_{\text {advection }}+\underbrace{\frac{\partial\left(v_{p, i} c_{i}\right)}{\partial z}}_{\text {segregation }}-\underbrace{\left[\frac{\partial}{\partial x}\left(D \frac{\partial c_{i}}{\partial x}\right)+\frac{\partial}{\partial z}\left(D \frac{\partial c_{i}}{\partial z}\right)\right]}_{\text {diffusion }}=0
$$

where $c_{i}$ is the concentration of species $i, u$ and $w$ are the mean granular velocity in the streamwise $(x)$ and surface-normal $(z)$ directions, respectively (note that this coordinate system is different than that for the experiments). The first term in the equation is the rate of change of species concentration $c_{i}$, the second and third terms account for advection due to mean flow, the fourth term accounts for segregation due to the species specific percolation velocity $v_{p, i}$, which is the component normal to the free surface, and the bracketed terms account for collisional diffusion. A key aspect of this bidisperse model is the dependence of the percolation velocity of species $i$ on the local shear rate and the local concentration of the other particle species $[12,15]$.

Here we apply this model using conditions for fullydeveloped steady flow on a quasi-2D bounded heap [16], but instantaneously change the velocity field (and thereby the shear rate) and flowing layer thickness from that for a low flow rate to that for a high flow rate and vice versa. In this way, the deposition of particles corresponding to the modulated feed flow rate (alternating between low to high) can be modeled, though this approach does not account for the propagation of the flow rate change along the length of the flowing layer evident in Fig. 2. Nevertheless, it provides a first order approximation of the effect of a modulated feed flow rate on the particle segregation and subsequent deposition on the heap, which is expected to be valid when the switching time between different flow rates is long compared to the relaxation time.

Fig. 4 compares experiments (extracted from an image like that in Fig. 1) and model results calculated using an operator splitting approach $[12,17]$. In the operator spliting approach at each time step the 
advection step is solved with a matrix mapping method [18], and the segregation/diffusion step is solved with an implicit Crank-Nicolson method. It is clear that even the simple application of the model (alternating between high and low flow rates) represents the stratification in the experiments quite well, particularly in terms of the wavelength of the stratified layers. Work to refine this model is currently under way, primarily focused on the non-uniform local deposition rate.

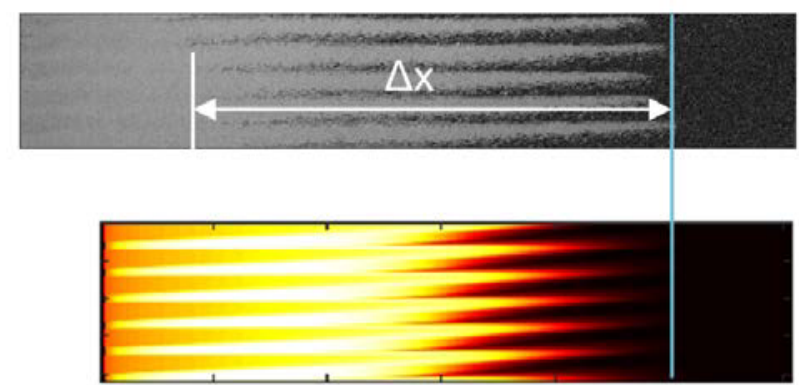

Fig. 4: Comparison of concentration fields from experiment (upper) and continuum model solved with steady state parameters for bidisperse modulated bounded heap flow (lower). Experiments used $0.05 \mathrm{~cm}$ (light) and $0.2 \mathrm{~cm}$ (dark) particles. Modulated feed flow conditions: $q_{f}=23.6 \mathrm{~cm}^{2} / \mathrm{s}$ for $t_{f}=5 \mathrm{~s}$ and $q_{s}=2.0 \mathrm{~cm}^{2} / \mathrm{s}$ for $t_{s}=20 \mathrm{~s}$. The extent of the model domain is reduced on the left to account for feed zone effects in the experiment.

\section{Summary}

Modulation of the feed flow rate can be used to force a stratified segregation pattern for a size bidisperse material in bounded heap flow. This may have significant advantages over the usual fully segregated pattern that otherwise occurs. For instance, if the bounded heap is formed in a hopper that is to be emptied later, a stratified segregation pattern will be mixed as it exits the hopper, whereas a fully segregated pattern will result in smaller particles exiting before larger particles exit.

Of course, further work is necessary to fully characterize the impact of the feed flow parameters (high and low flow rate magnitudes and durations) so they can be optimized for stratification. A better understanding of the mechanism described briefly with respect to Fig. 3 would aid in further optimizing the parameters for stratification. And the advection-diffusion-segregation model can be adapted to account for the propagation of the change in the flow rate down the heap, rather than the simple first order approach demonstrated in Fig. 4. Nevertheless, these results demonstrate the potential for modelling feed flow modulation and using it to intentionally generate stratified patterns in segregating granular flows.

The authors gratefully acknowledge the support of the US National Science Foundation, Grant No. CBET1511450 .

\section{References}

1. S.W. Meier, R.M. Lueptow, J.M. Ottino, Adv. Phys. 56, 757 (2007).

2. I.S. Aranson, L.S. Tsimring, Rev. Modern Phys., 78, 641 (2006).

3. S.B. Savage, C.K.K. Lun, J. Fluid Mech., 189, 311 (1988).

4. J.C. Williams, Powder Tech., 2, 13-20 (1968).

5. H.A. Makse, S. Havlin, P.R. King, H.E. Stanley, Nature 386, 379 (1997).

6. J.M.N.T. Gray and K. Hutter, Cont. Mech. Thermodyn. 9, 341 (1997).

7. Y. Fan, Y. Boukerkour, T. Blanc, P.B. Umbanhowar, J.M. Ottino, R.M. Lueptow, Phys. Rev. E 86, 051305 (2012).

8. O. Pouliquen, J.W. Vallance, Chaos 9, 621 (1999).

9. J.M.N.T. Gray and C. Ancey, J. Fluid Mech. 629, 387 (2009).

10. J.M.N.T. Gray and V.A. Chuganov, J. Fluid Mech. 569, 365 (2006).

11. A.R. Thornton, J.M.N.T. Gray, A.J. Hogg, J. Fluid Mech. 550, 1 (2006).

12. Y. Fan, C.P. Schlick, P.B. Umbanhowar, J.M. Ottino, R.M. Lueptow, J. Fluid Mech. 741, 252 (2014).

13. D.R. Tunuguntla, O. Bokhove, A.R. Thornton, J. Fluid Mech., 749, 99 (2014).

14. H. Xiao, P.B. Umbanhowar, J.M. Ottino, R.M. Lueptow, Proc. Royal Soc. A 472, 20150856 (2016).

15. C.P. Schlick, Y. Fan, A.B. Isner, P.B. Umbanhowar, J.M. Ottino, R.M. Lueptow, AIChE J. 61, 1524 (2015).

16. Y. Fan, P.B. Umbanhowar, J.M. Ottino, R.M. Lueptow, Proc. Royal Soc. A 469, 20130235 (2013).

17. C.P. Schlick, I.C. Christov, P.B. Umbanhowar, J.M. Ottino, R.M. Lueptow, Phys. Fluids 25, 10 (2013).

18. M.K. Singh, O.S. Galaktionov, H.E.H. Meijer, P.D. Anderson, Comput. Chem. Eng. 33, 8 (2009). 